\title{
Water molecular flow control with a $(5,5)$ nanocoil switch
}

\author{
Shin-Pon Ju • Jenn-Sen Lin · Jin-Yuan Hsieh • \\ Meng-Hsiung Weng $\cdot$ Ming-Chang Chen
}

Received: 10 April 2013/Accepted: 19 July 2013/Published online: 11 August 2013

(C) The Author(s) 2013. This article is published with open access at Springerlink.com

\begin{abstract}
Molecular dynamics simulation was employed to investigate the diffusion behaviors of water molecules within a $(5,5)$ carbon nanocoil $(\mathrm{CNC})$ at different tensile strains, the length and coil diameter of CNC are 22 and $6.83 \AA$ Á, respectively. Condensed-phase, optimized molecular potentials for atomistic simulation studies were employed to model the interaction between atoms. The results show that the diffusion in the axial direction can be enhanced by the tensile strain and the water molecule flow can be blocked at a higher strain once the deformed areas appear at the higher strain. Moreover, the deformed $(5,5) \mathrm{CNC}$ at strain of $2.8 \mathrm{can}$ recover its original structure at strain of 0 , indicating that the adjustment of diffusion coefficient is repeatable by applying different strains in the axial direction.
\end{abstract}

Keywords Nanocoil - Molecular dynamics . Water molecule $\cdot$ Diffusion coefficient

S.-P. Ju $(\bowtie) \cdot$ M.-H. Weng · M.-C. Chen

Department of Mechanical and Electro-Mechanical

Engineering, National Sun Yat-sen University,

Kaohsiung 804, Taiwan, Republic of China

e-mail: jushin-pon@mail.nsysu.edu.tw

J.-S. Lin

Department of Mechanical Engineering, National United

University, Miao-Li 360, Taiwan, Republic of China

J.-Y. Hsieh

Department of Mechanical Engineering, Minghsin Institute of Technology, Hsin-Chu 304, Taiwan,

Republic of China

\section{Introduction}

Since carbon material is strongly hydrophobic to water (Müller et al. 1996; Martin and Kohli 2003), carbon nanotubes (CNTs) have attracted great interest for use in biological applications (Zhao et al. 2012; Bianco et al. 2005). Among those CNTs, carbon nanocoils (CNCs) are the most notable because of their 3D helical structure (Chen et al. 2003). CNCs possess high tensile strength and superelasticity (Lau et al. 2006; Liu et al. 2010), and thus they have great potential applications as nanomechanical and electromagnetic device components, such as nanosprings (Williams et al. 2003), oscillators (Papadakis et al. 2004), and nanoelastic memory (Changa and Park 2006). These outstanding mechanical properties could be also instrumental in developing a novel efficient functional nanofluidic switch for controlling the flow amount of molecules from one end to the other end of CNCs.

Many experimental and theoretical investigations have demonstrated that water molecules in a nanoscale environment show properties different from those of the molecules in a bulk system (Naguib et al. 2004; Rossi et al. 2004; Byl et al. 2006). Koga et al. (2001) found that structural and transport properties of water molecules are strongly affected by the nanoconfinement effect. Holt et al. (2006) reported that a high flow rate of water occurs when it crosses through CNT pore membranes with diameters less than $2 \mathrm{~nm}$. Thomas et al. (2010) examined the pressure-driven water flow through CNTs of different radii. They found that the 
viscosity of water is not affected when the radius is larger than $10 \mathrm{~nm}$. Zheng et al. (2005) employed molecular dynamics (MD) simulation to investigate the transport of water and methanol in hydrophobic single-walled nanotubes with various diameters. They found that hydrogen bonding plays a critical role for fluid transport across the pore.

The transport control of water in a confined space by different strain mechanisms has been widely theoretically investigated. Lu et al. (2012) employed MD simulations to investigate the flux of water inside a deformed CNT driven by an oscillating charge. Their results indicate that the oscillating frequency of the charge strongly influences the flux. Li et al. (2008) studied the structures of water confined in nanotubes undergoing axial tensile strain, and found a tendency in the water molecular structure to form chains. Fang et al. (2008) investigated the behavior of water across single-walled CNTs under different external forces on nanotube wall. Their results show that single-walled CNTs can be used as a nanoscale switch to control the water flux by the deformation of nanotube wall.

These studies demonstrate how MD simulation is a powerful tool for the investigation of structural behavior at an atomic level. In this study, we conduct MD simulations to study the diffusion behaviors of water molecules inside a $(5,5) \mathrm{CNC}$ during axial tension. Since the water molecules diffuse generally across cell membranes in very low numbers, this research investigates controlling the diffusion of ultra-low water flow between cells or membranes. In this study, we found that the flow of molecules is interrupted as tensile strain is applied between the two ends of the CNC because of deformations. This study provides the possibility of an excellent on-off switch application.

\section{Simulation detail}

MD methods, implemented in the DISCOVER package (Rigby 2004; Accelrys Software Inc. 2012), were used to study the diffusion behavior of water molecules within the $(5,5) \mathrm{CNC}$ at different tensile strains.

MD is the principal tool to understand the dynamic behaviors of individual atoms and fine structure. MD simulations method is based on the classical Newtonian equations of motion (Eq. (1)) for all atoms in the system. The result shows the trajectory that specifies how the positions and velocities of the particles in the system alter with time. Hence, the property of the system or molecule can be evaluated by the average of trajectories.

$m_{i} \frac{\mathrm{d}^{2} r_{i}(t)}{\mathrm{d} t^{2}}=F_{i}(t)=-\frac{\partial U\left(r_{i}\right)}{\partial r_{i}}$,

where $m_{i}, r_{i}$, and $F_{i}$ are the mass, position vector, force vector of molecule $i$, respectively. $U\left(r_{i}\right)$ is the force field.

In this simulation, the condensed-phase, optimized molecular potential for atomistic simulation studies (COMPASS) potential was employed to model the interatomic forces between the carbon atoms, between water molecules, and between water molecules and carbon atoms. The COMPASS potential $U_{\text {total }}$ can be expressed as (Sun et al. 1998)

$$
\begin{aligned}
U_{\text {total }}=U_{b} & +U_{\theta}+U_{\phi}+U_{\chi}+U_{b b^{\prime}}+U_{b \theta} \\
& +U_{b \phi}+U_{\theta \theta^{\prime}}+U_{\theta \theta^{\prime} \phi}+U_{\text {else }}+U_{\mathrm{LJ}}
\end{aligned}
$$

$$
\begin{aligned}
U_{\text {total }}= & \sum_{b}\left[k_{2}\left(b-b_{0}\right)^{2}+k_{3}\left(b-b_{0}\right)^{3}+k_{4}\left(b-b_{0}\right)^{4}\right] \\
& +\sum_{\theta}\left[H_{2}\left(\theta-\theta_{0}\right)^{2}+H_{3}\left(\theta-\theta_{0}\right)^{3}\right. \\
& \left.+H_{4}\left(\theta-\theta_{0}\right)^{4}\right] \\
& +\sum_{\phi}\left[V_{1}(1-\cos \phi)+V_{2}(1-\cos 2 \phi)\right. \\
& \left.+V_{3}(1-\cos 3 \phi)\right] \\
& +\sum_{x} k_{2} \chi^{2}+\sum_{b} \sum_{b^{\prime}} k_{b b^{\prime}}\left(b-b_{0}\right)\left(b^{\prime}-b_{0}^{\prime}\right) \\
& +\sum_{b} \sum_{\theta} K_{b \theta}\left(b-b_{0}\right)\left(\theta-\theta_{0}\right) \\
& +\sum_{b} \sum_{\phi}\left(b-b_{0}\right) \\
& \times\left[k_{1 b \phi} \cos \phi+k_{2 b \phi} \cos 2 \phi+k_{3 b \phi} \cos 3 \phi\right] \\
& +\sum_{\theta} \sum_{\phi}\left(\theta-\theta_{0}\right) \\
& \times\left[k_{1 \theta \phi} \cos \phi+k_{2 \theta \phi} \cos 2 \phi+k_{3 \theta \phi} \cos 3 \phi\right] \\
& +\sum_{b} \sum_{\theta^{\prime}} k_{\theta \theta^{\prime}}\left(\theta-\theta_{0}\right)\left(\theta^{\prime}-\theta_{0}^{1}\right) \\
& +\sum_{\theta} \sum_{\theta^{\prime}} \sum_{\phi} k_{\theta \theta^{\prime} \phi}\left(\theta-\theta_{0}\right)\left(\theta^{\prime}-\theta_{0}^{\prime}\right) \cos \phi \\
& +\sum_{i>j} \varepsilon_{i j}\left[2\left(\frac{\sigma}{r i j}\right)^{9}-3\left(\frac{\sigma}{r_{i j}}\right)^{6}\right]+\sum_{i>j} \frac{q_{i} q_{j}}{r_{i j}}, \\
&
\end{aligned}
$$


where potentials $U_{b}, U_{\theta}, U_{\phi}$, and $U_{\chi}$ are the quartic polynomials for the bond, angle, torsion, and the out-ofplane angle coordinate, respectively. Terms 5-9 of ( $U_{b b^{\prime}}$, $U_{b \theta}, U_{b \phi}, U_{\theta \theta^{\prime}}$, and $U_{\theta \theta^{\prime} \phi}$ ) of Eq. (2) represent the crosscoupling terms. $U_{\text {else }}$ and $U_{\mathrm{LJ}}$ represent the Coulombic interaction between the atomic charges and van der Waals interactions. In this present simulation model, the water molecules were initially set inside the $(5,5) \mathrm{CNC}$ randomly. Figure 1 presents a simulation model of water molecules inside the periodic $(5,5) \mathrm{CNC}$ with different numbers of water molecules: 72,123 , and 179 . The $(5,5) \mathrm{CNC}$ is composed of 2,580 carbon atoms, and its pitch length and coil diameter are 22 and $6.83 \AA$, respectively. The 7-carbon and 5-carbon rings are located at the vertices of the hexagonal inner and outer walls of the $(5,5)$ nanocoil, respectively. The segments of $(5,5)$ CNTs are connected by these vertex portions to form a stable $(5,5)$ nanocoils with the spiral structure. For the other CNCs, they are designated as the same indexes of the corresponding CNT segments of CNCs. In the axial tensile process, the $(5,5) \mathrm{CNC}$ still retains periodicity. The system temperatures are assigned a constant $300 \mathrm{~K}$. The Andersen thermostat (Andersen 1980; Weinan and Li 2008) is applied to insure that the temperature of the system remains constant during simulation, and the Verlet algorithm (Verlet 1967; Swope et al. 1982) is employed to calculate the trajectories of the atoms. Moreover, all the carbon atoms of the $\mathrm{CNC}$ are fully relaxed during the simulation. In this study, we chose different elongated structures of strains to investigate the diffusion properties of water molecules within CNC. After $\mathrm{CNC}$ is strengthened, the structures are relaxed once again. The total relaxation time is set to $200 \mathrm{ps,}$ because such a long equilibrium time can insure that the elongated $(5,5) \mathrm{CNC}$ is relaxed. The data collection and analyses were performed on the final 100 ps.

\section{Results and discussion}

The $z$ or axial direction is defined as the tensile direction shown in the side view of $(5,5) \mathrm{CNC}$ in Fig. 1, and the two different local structures are defined as the vertex and CNT parts. Figure 2 shows the mean-square displacement plots in the $z$ dimension $\left(\mathrm{MSD}_{z}\right)$ of water molecules within the $(5,5) \mathrm{CNT}$, and CNC at strain of 0 . The $\mathrm{MSD}_{z}$ is defined as

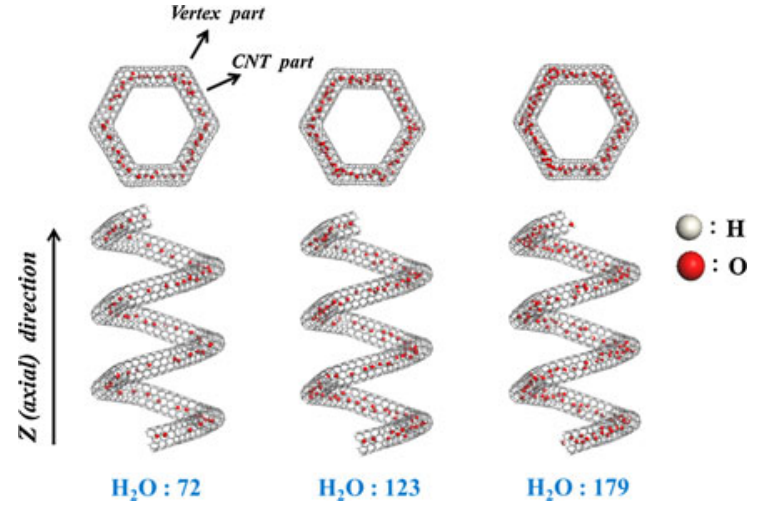

Fig. 1 Simulation model of water molecules through the $(5,5)$ CNC with different numbers of water molecules, 72, 123, and 179

$\operatorname{MSD}_{z}=\left\langle\sum_{i}^{N}\left[r_{z i}(t)-r_{z i}\left(t_{0}\right)\right]^{2} / N\right\rangle$,

where $r_{z i}(t)$ represents the $z$ coordinate of the oxygen atom of water molecule $i$ at delay time $t$, and $r_{z i}\left(t_{0}\right)$ indicates the referenced $z$ coordinate at referenced time $t_{0} ; N$ represents the total number of water molecules within CNT or CNC. The brackets are interpreted as average over time origins. The diffusion of water molecules was also examined by calculating a self-diffusion coefficient $D$ in the $z$ direction $\left(D_{z}\right)$ from $\mathrm{MSD}_{z}$ over the course of simulation. The self-diffusion coefficient is obtained from the $\mathrm{MSD}_{z}$ via the Einstein equation (Meunier 2005) which is rewritten as

$D_{z}=\frac{1}{2 N} \lim _{t \rightarrow \infty} \frac{\mathrm{d}}{\mathrm{d} t}\left\langle\sum_{i}^{N}\left[r_{z i}(t)-r_{z i}(0)\right]^{2}\right\rangle$.

The diffusion coefficient of bulk water at $300 \mathrm{~K}$ was calculated first by Eq. (5), with the value of $2.35 \times 10^{-9} \mathrm{~m}^{2} / \mathrm{s}$ being very close to the experimental value of $2.3 \times 10^{-9} \mathrm{~m}^{2} / \mathrm{s}$ (Mills 1973) indicating the reliability of the COMPASS force field to predict the diffusion behavior of water molecules. In Fig. 2, $\mathrm{MSD}_{z}$ profiles of the $(5,5) \mathrm{CNT}$ and $(5,5) \mathrm{CNC}$ averaged over $100 \mathrm{ps}$ (after the first $100 \mathrm{ps}$ to attain equilibrium) are displayed. It shows a more significant rise in the number of water molecules within the $(5,5)$ $\mathrm{CNT}$, which indicates that water molecules have faster diffusion in the axial direction within the $(5,5) \mathrm{CNT}$ than within the $(5,5) \mathrm{CNC}$. This is because the majority of the water diffusion direction within a 


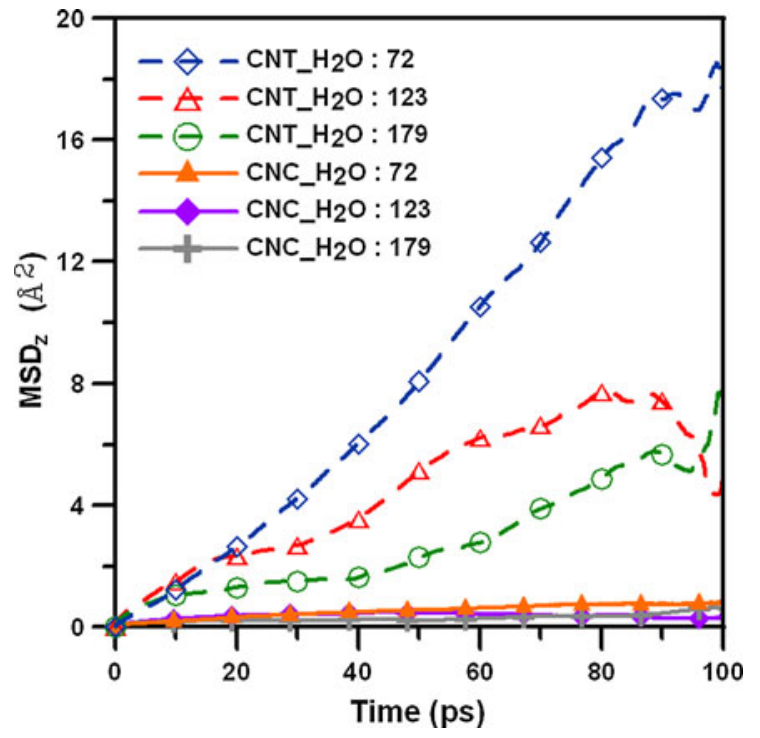

Fig. 2 The mean-square displacement plots in the $z$ dimension $\left(\mathrm{MSD}_{z}\right)$ of water molecules within the $(5,5) \mathrm{CNT}$ and $\mathrm{CNC}$ at strain of 0

$(5,5) \mathrm{CNT}$ is along the axial direction, and there is almost no diffusion in the plane normal to the CNT axis. For the $(5,5) \mathrm{CNC}$, the $\mathrm{MSD}_{z}$ profiles are much lower than those of $(5,5) \mathrm{CNTs}$, indicating the water diffusion within the $(5,5) \mathrm{CNCs}$ in the $z$ direction is much slower than that within $(5,5)$ CNTs. This is because water molecules within the $(5,5) \mathrm{CNC}$ has larger diffusion space around the $z$ direction (i.e., in $x-y$ plane).

Since CNCs have been proven to be a superelastic material which may still be intact at a very large tensile strain (Chen et al. 2003; Liu et al. 2010), it is possible to adjust the $D_{z}$ by applying different strains upon the $(5,5)$ CNC. Figure 3 shows the $D_{z}$ values for different water numbers within the $(5,5) \mathrm{CNC}$. $(5,5) \mathrm{CNCs}$ under six different strains, labeled as S1-S6 in Fig. 3, are considered. $D_{z}$ values first increase with the strain and then decrease after their maximal $D_{z}$ values. Generally speaking, the diffusion within the $(5,5)$ $\mathrm{CNC}$ with fewer water molecules is faster because there are fewer hydrogen bonds forming between water molecules.

The morphologies at different strains for different water numbers within the $(5,5) \mathrm{CNC}$ are shown in Fig. $4 \mathrm{a}-\mathrm{c}$, and the water chain structures at the first strains are also illustrated. In Fig. 4b, the water chain at $\mathrm{S} 1$ indicates that 123 water molecules are sufficient

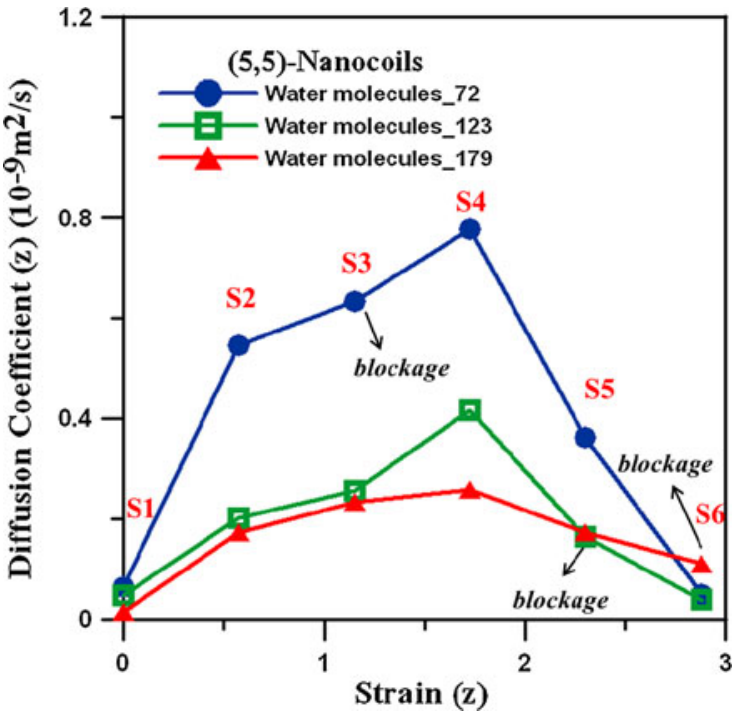

Fig. 3 Diffusion coefficient for different water numbers within the $(5,5) \mathrm{CNC}$ under different strains

to form a complete water chain within the $(5,5) \mathrm{CNC}$ and, for the system with 179 water molecules, it appears easier for water molecules to accumulate at the vertex parts, as shown in Fig. 4c. For the case of 123 water molecules, several shorter water chains can be seen in Fig. 4a because the water number is not sufficient, and the hydrogen bond between water molecules aids in the water molecules aggregating in a chainlike form. In Fig. $4 \mathrm{a}-\mathrm{c}$, it is clear the segments of $(5,5)$ CNT parts are aligned more along the $z$ direction with continuously increasing strain. When the strain becomes larger, some deformed areas appear, indicated by arrows in Fig. 4a-c, resulting in a blockage of water molecules within the $(5,5) \mathrm{CNC}$. The strains at which the blockage occurs are at S3, S5, and S6 for cases of 72, 123, and 179 water molecules, respectively. Because the $(5,5) \mathrm{CNC}$ is hydrophobic, the repulsive interaction between water molecules, and the $(5,5) \mathrm{CNC}$ wall is relatively stronger than that between other nonpolar molecules and the CNC wall. Consequently, the higher water density within the $(5,5) \mathrm{CNC}$ helps in preventing deformation. In Fig. 4a, $\mathrm{b}$, it is clear that more deformed areas will appear when the strain becomes larger, with the water molecules becoming distributed in different segments formed by the deformed areas. For $D_{z}$ values in Fig. 3, the strains at which the deformations appear are indicated as "blockage" for three different water 
(a)

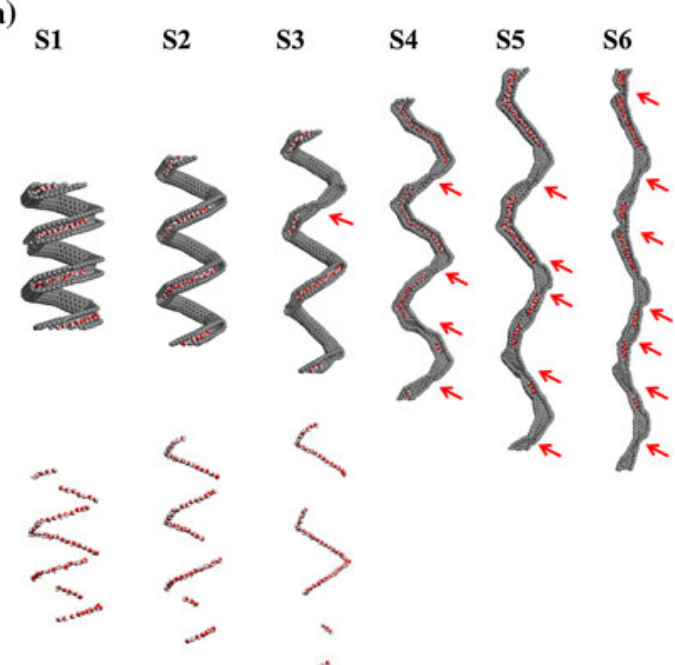

(b)

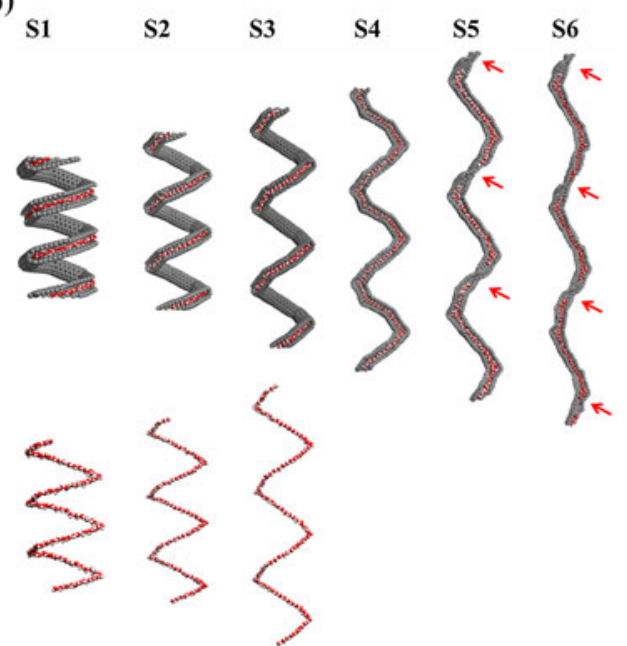

(c)

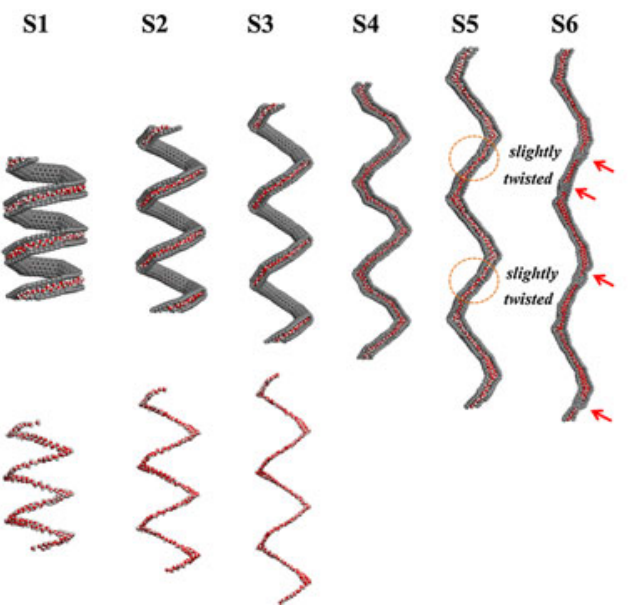

Fig. 4 The morphologies at different strains for simulation of a 72 , b 123 , and c 179 water molecules within the $(5,5) \mathrm{CNC}$ densities, indicating that the $\mathrm{CNC}$ channels are no longer open after those points: that is, after $\mathrm{S} 3$ for 72 molecules, S5 for 123 molecules, and S6 for 179 molecules. For the case of 72 water molecules, $D_{z}$ still increases at S4 although one deformed area has appeared at $\mathrm{S} 3$. The reason for the diffusion coefficient of S4 being larger than S3 is because water molecules are locked locally within the segments created by deformations, and therefore the local water diffusion within the segments in the $z$ direction is higher. As the strain increases to S5 and S6, more deformed areas appear, and the local densities at different segments become much higher than those at $\mathrm{S} 1$ such that there is not enough space for water diffusion, and the diffusion coefficients in the $z$ direction decrease. For the case of 123 water molecules, the number of water molecules is sufficient to form a water chain within the $(5,5)$ CNC. In Fig. 3, one can see that $D_{z}$ for 123 water molecules significantly increases from $0.047 \times 10^{-9}$ to $0.417 \times 10^{-9} \mathrm{~m}^{2} / \mathrm{s}$ (about an 8.9-fold increase) at strains from S1 to S4. Once the deformed areas appear, $D_{z}$ values will decrease significantly with increasing strain. For the case of 179 water molecules, the deformed areas appear at S6, shown in Fig. 4c, but $D_{z}$ begins to decrease when strain is larger than S4. Several slightly twisted areas at the $(5,5)$ CNT segments can be seen in Fig. 4c, which makes the cross sections slightly narrower than those at strain 4 , leading to more water molecules being blocked at the vertex parts at strain S5.

In order to determine whether the deformed $(5,5)$ $\mathrm{CNC}$ at higher strain can recover its intact structure at strain of 0 (or stress 0 in the tensile direction), the case of 72 water molecule $(5,5) \mathrm{CNC}$ structure at strain S6 is used in the isobaric-isothermal ensemble (NPT). The Parrinello algorithm (Parrinello and Rahman 1981) is used to relax the stress at strain S6 to $1 \mathrm{~atm}$, and the stresses in the $x$ and $y$ dimensions are set to 0 during the simulation. Six snapshots at sequential time steps are illustrated in Fig. 5, and it is clear that the deformed $(5,5) \mathrm{CNC}$ will recover the $\mathrm{S} 1$ structure, and the water molecules can flow throughout the $(5,5) \mathrm{CNC}$ again. A closer observation of the deformed parts at 0 ps shows that there are two different deformation types for the $(5,5) \mathrm{CNC}$ : twisted and necking parts. The twisted parts occur at the $(5,5)$ CNT segments while the necking parts appear at the vertex. The local structural variations for one twisted and one necking part deformation $(5,5)$ $\mathrm{CNC}$ are also shown and detailed in Fig. 5 and its insets. 
Fig. 5 Six snapshots at sequential time steps showing local structural variations at one twisted and one necking part deformation in $(5,5) \mathrm{CNC}$
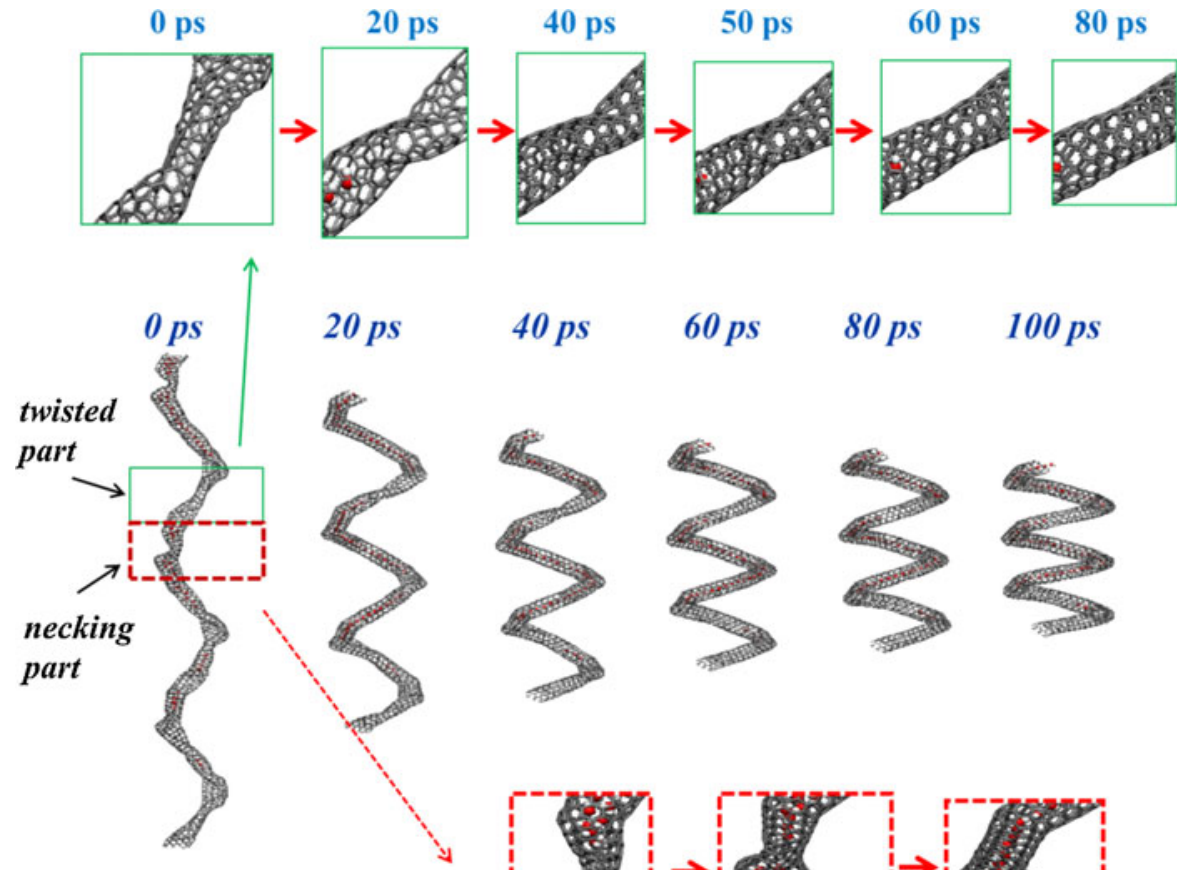

$40 \mathrm{ps}$

$60 \mathrm{ps} \quad 80 \mathrm{ps}$

$100 \mathrm{ps}$
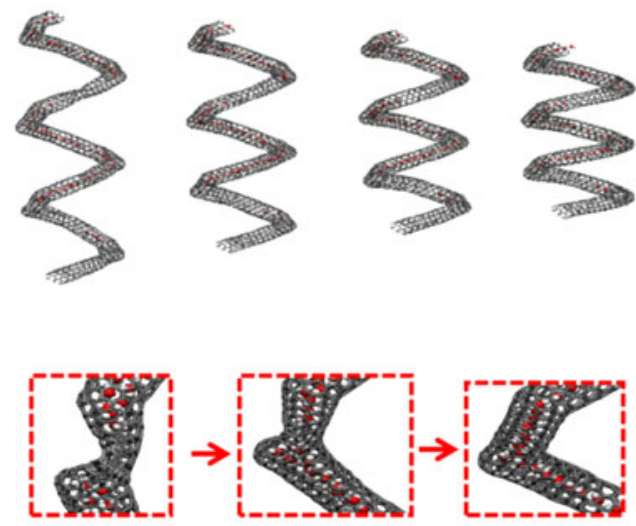

0 ps

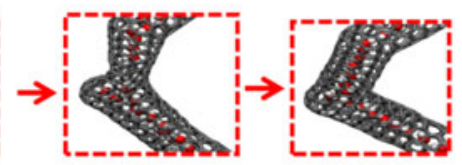

$10 \mathrm{ps}$

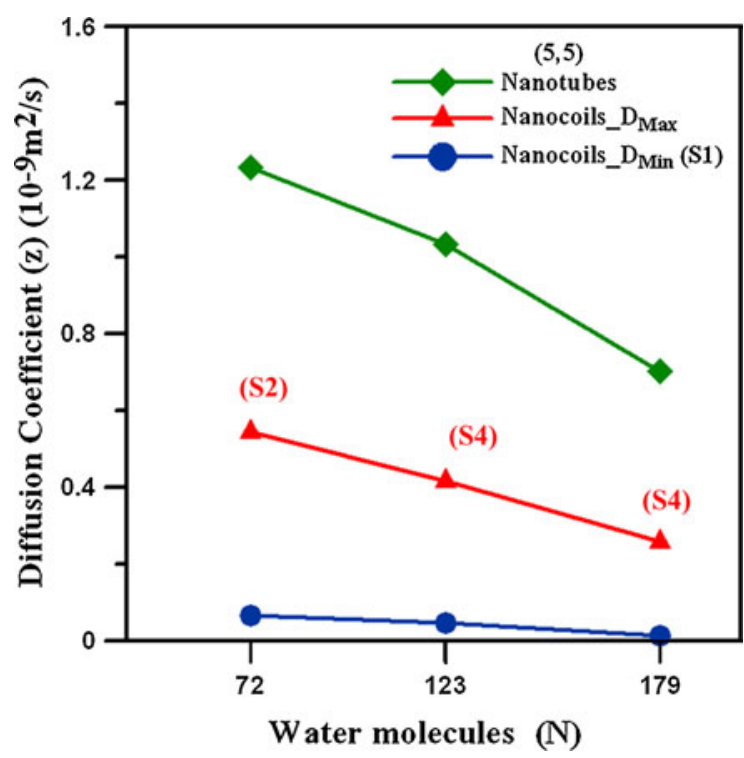

Fig. $6 D_{z}$ values for systems of different numbers of water molecules within the $(5,5) \mathrm{CNT}$ and $(5,5) \mathrm{CNC}$

The twisted part completely recovers to the $(5,5) \mathrm{CNT}$ segment after $80 \mathrm{ps}$, and the necking part completely recovers to the vertex part at strain $\mathrm{S} 1$. This reveals that the $(5,5) \mathrm{CNC}$ can repeatedly be used as a water molecule switch by the generation of deformed areas under tensile strain and subsequent structural recovery from the relaxation of tensile strain.

\section{Conclusion}

This study examines the diffusion behaviors of water molecules within the $(5,5) \mathrm{CNC}$ at different strains by MD simulation. The diffusion in the axial direction can be enhanced by the tensile strain, and the water molecule flow can be blocked at higher strain, after the deformed areas appear. Figure 6 summarizes the $D_{z}$ values for systems of different numbers of water molecules within the $(5,5) \mathrm{CNT}$ and $(5,5) \mathrm{CNC}$. $D_{\mathrm{Max}}$ is the largest $D_{z}$ without blockage, and $D_{\mathrm{Min}}$ is the lowest value at strain $\mathrm{S} 1$ for each case. By applying the tensile strain, the $D_{z}$ value can be enhanced from $0.065 \times 10^{-9}$ to $0.545 \times 10^{-9} \mathrm{~m}^{2} / \mathrm{s}$ for the case of 72 water molecules and increased from $0.014 \times 10^{-9}$ to $0.257 \times 10^{-9} \mathrm{~m}^{2} / \mathrm{s}$ for 179 water molecules. For the $(5,5) \mathrm{CNT}$, although the water diffusion in the axial direction is faster than that within the $(5,5) \mathrm{CNC}$, the adjustment of diffusion behavior by axial strain is not significant. As shown in this study, CNCs could be a promising material for the 
use as an artificial gated bio-channel. Due to their ability to control the flow amount of molecules from one end to the other end of CNCs by axial strain, the applications of $\mathrm{CNCs}$ on the nanosyringe, drug nanodelivery, or nanoswitch could be implemented in the future.

Open Access This article is distributed under the terms of the Creative Commons Attribution License which permits any use, distribution, and reproduction in any medium, provided the original author(s) and the source are credited.

\section{References}

Accelrys Software Inc. (2012) Discovery studio modeling environment. Release 3.5, Accelrys Software Inc., San Diego

Andersen HC (1980) Molecular dynamics simulations at constant pressure and/or temperature. J Chem Phys 72:2384-2393. doi:10.1063/1.439486

Bianco A, Kostarelos K, Prato M (2005) Applications of carbon nanotubes in drug delivery. Curr Opin Chem Biol 9:674-679. doi:10.1016/j.cbpa.2005.10.005

Byl O, Liu JC, Wang Y, Yim WL, Johnson JK, Yates JT (2006) Unusual hydrogen bonding in water-filled carbon nanotubes. J Am Chem Soc 128:12090-12097. doi:10.1021/ ja057856u

Changa JH, Park W (2006) Nano elastic memory using carbon nanocoils. Joo Han Chang Wanjun Park 3:30-35

Chen X, Zhang S, Dikin DA, Ding W, Ruoff RS (2003) Mechanics of a carbon nanocoil. Nano Lett 3:1299-1304. doi: $10.1021 / \mathrm{nl} 034367 \mathrm{o}$

Fang HP, Wan RZ, Gong XJ, Lu HJ, Li SY (2008) Dynamics of single-file water chains inside nanoscale channels: physics, biological significance and applications. J Phys D 41:103002. doi:10.1088/0022-3727/41/10/103002

Holt JK, Park HG, Wang YM, Stadermann M, Artyukhin AB, Grigoropoulos CP, Noy A, Bakajin O (2006) Fast mass transport through sub-2-nanometer carbon nanotubes. Science 312:1034-1037. doi:10.1126/science.1126298

Koga K, Gao GT, Tanaka H, Zeng XC (2001) Formation of ordered ice nanotubes inside carbon nanotubes. Nature 412. doi:10.1038/35090532

Lau K, Lu M, Liao K (2006) Improved mechanical properties of coiled carbon nanotubes reinforced epoxy nanocomposites. Composites A 37:1837-1840. doi:10.1016/j.compositesa. 2005.09.019

Li H, Zhang XQ, Liew KM, Liu XF (2008) Structures of water molecular nanotube induced by axial tensile strains. Phys Lett A 372:6288-6293. doi:10.1016/j.physleta.2008.08.038

Liu LZ, Gao HL, Zhao JJ, Lu JP (2010) Superelasticity of carbon nanocoils from atomistic quantum simulations. Nanoscale Res Lett 5:478-483. doi:10.1007/s11671-010-9545-X

Lu H, Nie XC, Wu FM, Zhou XY, Kou JL, Xu YS, Liu Y (2012) Controllable transport of water through nanochannel by rachet-like mechanism. J Chem Phys 136:174511. doi:10. 1063/1.4707744

Martin CR, Kohli P (2003) The emerging field of nanotube biotechnology. Nat Rev Drug Discov 2:29-37. doi:10.1038/ $\operatorname{nrd988}$
Meunier M (2005) Diffusion coefficients of small gas molecules in amorphous cis-1,4-polybutadiene estimated by molecular dynamics simulations. J Chem Phys 123:134906134907. doi:10.1063/1.2049274

Mills R (1973) Self-diffusion in normal and heavy water in the range 1-45 deg. J Phys Chem 77:685-688. doi:10.1021/ j100624a025

Müller EA, Rull FL, Vega LF, Gubbins KE (1996) Adsorption of water on activated carbons: a molecular simulation study. J Phys Chem 100:1189-1196. doi:10.1021/jp952233w

Naguib N, Ye H, Gogotsi Y, Yazicioglu AG, Megaridis CM, Yoshimur M (2004) Observation of water confined in nanometer channels of closed carbon nanotubes. Nano Lett 4:2237-2243. doi:10.1021/n10484907

Papadakis SJ, Hall AR, Williams PA, Vicci L, Falvo MR, Superfine R, Washburn S (2004) Resonant oscillators with carbon-nanotube torsion springs. Phys Rev Lett 93:146101. doi:10.1103/PhysRevLett.93.146101

Parrinello M, Rahman A (1981) Polymorphic transitions in single crystals: a new molecular dynamics method. J Appl Phys 52:7182-7190. doi:10.1063/1.328693

Rigby D (2004) Fluid density predictions using the COMPASS force field. Fluid Phase Equilib 217:77-87. doi:10.1016/j. fluid.2003.08.019

Rossi MP, Ye HH, Gogotsi Y, Babu S, Ndungu P, Bradley JC (2004) Environmental scanning electron microscopy study of water in carbon nanopipes. Nano Lett 4:989-993. doi: $10.1021 / \mathrm{n} 1049688 \mathrm{u}$

Sun H, Ren P, Fried JR (1998) The COMPASS force field: parameterization and validation for phosphazenes. Comput Theor Polym Sci 8:229-246. doi:10.1016/S1089-3156(98) 00042-7

Swope WC, Andersen HC, Berens PH, Wilson KR (1982) A computer simulation method for the calculation of equilibrium constants for the formation of physical clusters of molecules: application to small water clusters. J Chem Phys 76:637-649. doi:10.1063/1.442716

Thomas JA, McGaughey AJH, Kuter-Arnebeck O (2010) Pressure-driven water flow through carbon nanotubes: insights from molecular dynamics simulation. Int $\mathrm{J}$ Therm Sci 49:281-289. doi:10.1016/j.ijthermalsci.2009.07.008

Verlet L (1967) Computer "Experiments" on classical fluids. I. Thermodynamical properties of Lennard-Jones molecules. Phys Rev 159:98-103. doi:10.1103/PhysRev.159.98

Weinan E, Dong L (2008) The Andersen thermostat in molecular dynamics. Commun Pure Appl Math 61:96-136. doi:10.1002/cpa.20198

Williams PA, Papadakis SJ, Patel AM, Falvo MR, Washburn S, Superfine R (2003) Fabrication of nanometer-scale mechanical devices incorporating individual multiwalled carbon nanotubes as torsional springs. Appl Phys Lett 82:805-807. doi:10.1063/1.1538346

Zhao H, Rizal B, McMahon G, Wang H, Dhakal P, Kirkpatrick T, Ren Z, Chiles TC, Naughton MJ, Cai D (2012) Ultrasensitive chemical detection using a nanocoax sensor. ACS Nano 6:3171-3178. doi:10.1021/nn205036e

Zheng J, Lennon EM, Tsao HK, Sheng YJ, Jiang SY (2005) Transport of a liquid water and methanol mixture through carbon nanotubes under a chemical potential gradient. J Chem Phys 122:214702. doi:10.1063/1.1908619 